\title{
Correlation Photoacoustic Measurement by Excitation with Synchrotron Radiation
}

\author{
Kenji KaTo* and Yoshinori SugrtanI** \\ *National Chemical Laboratory for Industry, Tsukuba, Ibaraki, 305 \\ **Department of Chemistry, The University of Tsukuba, Tsukuba, Ibaraki 305
}

\begin{abstract}
Correlation photoacoustic spectroscopy was carried out in X-ray region using synchrotron radiation(SR) as an exciting source. Thermal diffusion in copper foils and in laminated samples was investigated. The characteristic rise and fall times of correlation spectra, $\tau_{0}$ and $\tau_{t}$, respectively, were found to be influenced by sample thickness with the maximum values at intermediate thicknesses $(10$ and $50 \mu \mathrm{m})$ for copper samples. This was interpreted as arising from the competing effect between the thermal diffusion within the sample and the thermal radiation from the sample surface. For the laminated samples, increases of $\tau_{0}$ and $\tau_{f}$ were observed with the increase of thickness of polymer layer on the copper substrate.
\end{abstract}

Keywords Correlation, photoacoustic spectroscopy, X-ray, synchrotron radiation, thermal diffusion

Photoacoustic measurement provides information about the thermal properties of bulk and layered materials through non-destructive observation. ${ }^{1}$ So far, photoacoustic spectroscopy was mostly conducted in the spectral regions of UV through IR. It has been reported recently that the photoacoustic measurement in $\mathrm{X}$-ray region was successfully conducted using conventional X-ray tube ${ }^{2-4}$ and synchrotron radiation ${ }^{5}$ as exciting sources. In the photoacoustic study in Xray region, elemental analysis would be possible using the characteristic X-ray absorption for each element. For this purpose, the synchrotron radiation is an excellent X-ray source in terms of wide selectivity of wavelength, strong intensity, and directional nature of its beam. Therefore, photoacoustic measurement in Xray region by synchrotron radiation will be a new analytical tool for investigation in materials science. In addition, introduction of cross correlation technique into photoacoustic measurement provides further information about materials. This technique, correlation photoacoustics, has two features. One is the measurement of an impulse response curve, and the other is the measurement of the time resolved spectra of sample. These are referred to as the first and second kinds of correlation spectra, respectively. ${ }^{6}$ The former can give more detailed information of thermal properties and structure of layers ${ }^{7,8}$, particles, and subsurface defects than the conventional photoacoustic method which uses a regular interval modulating system. The latter gives photoacoustic spectra of deep levels of the sample, 6,8 and gives three-dimensional features of materials. The present paper reports the first attempt of correlation photoacoustic measurement of copper foil and layered samples in the X-ray region.

\section{Experimental}

Figure 1 shows the schematic diagram of the experimental arrangement. The experiment was made at the beam lines BL4A and BL15A in Photon Factory (National Laboratory for High Energy Physics, KEK, Tsukuba). X-Ray beams were monochromated to 1.37 $\AA$ (BL4A), $1.48 \AA$ (BL15A) and were focused to 3 $\mathrm{mm} \times 2 \mathrm{~mm}$ area on the samples. A mechanical random chopper containing two M-series $(n=31)$, pseud random binary sequence (PRBS), was used to modulate the beam. Chopper speed was varied from 1 to 8 rps (rotation per second). Figure 2 shows the photoacoustic cell used. The cell was made of brass

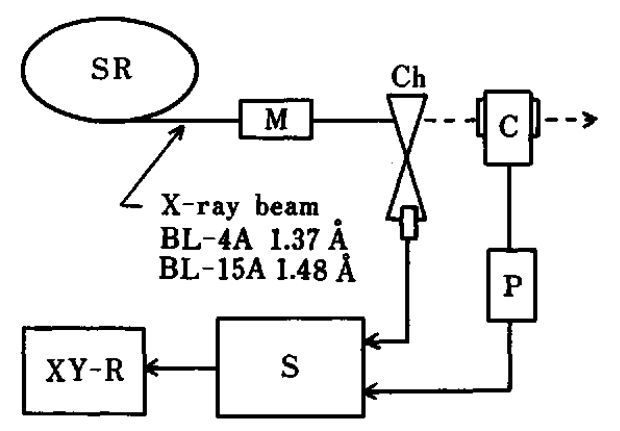

Fig. 1 Schematic diagram of the experimental arrangement. SR, synchrotron radiation source; $M$, monochromater; $\mathrm{Ch}, \mathrm{M}$-series random chopper; $\mathrm{C}$, photoacoustic cell; $P$, preamplifier; $S$, signal processor; $X Y-R, X Y$-recorder. 


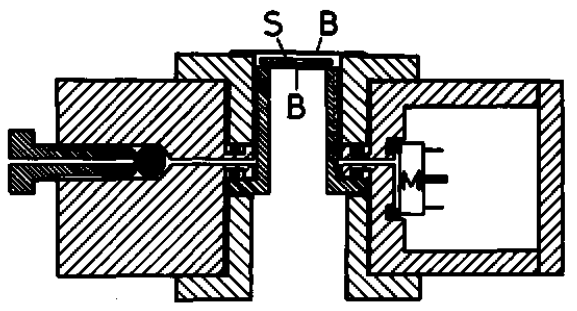

Fig. 2 Cross section of photoacoustic cell designed for the measurement in X-ray region. S, sample; $B$, beryllium windows; $\mathrm{M}$, condenser microphone; $\mathbf{O}$, O-rings.

and had beryllium windows $(10 \mathrm{~mm}$ diameter and 0.25 $\mathrm{mm}$ thick). The photoacoustic signal was detected by a condenser microphone and was preamplified at the beam site. The amplified signal was introduced into a signal analyzer (SM2100C, IWATSU Electric Co. Ltd.), where the cross correlation between photoacoustic signal and reference signal was calculated and was integrated 100 or 400 times. The reference signal was obtained at an optical coupler installed at the chopper. Sheets of copper foil (thickness 1 to $300 \mu \mathrm{m}$ and diameter 10 to $15 \mathrm{~mm}$ ) and laminated sheets of copper foils and adhesive tapes were used as samples. In the case of the latter samples, polymer adhesive tapes (thickness about $50 \mu \mathrm{m}$ ) were adhered on both sides of copper foil (thickness $100 \mu \mathrm{m}$, diameter $18 \mathrm{~mm}$ ). The signal intensities were normalized to the ring current.

\section{Results and Discussion}

Figure 3 shows the first kind correlation photoacoustic spectra of the copper foil with thickness of $10 \mu \mathrm{m}$ for 100 times integration. $\tau_{0}$ is defined as the time difference between the rising point and the top of the peak, while $\tau_{\mathrm{f}}$ is defined as that between the top and the half height point. Since the relaxation time from the excited levels is known to be negligibly short in the Xray region, $\tau_{0}$ and $\tau_{\mathrm{f}}$ are considered to reflect directly the thermal process of the sample in the photoacoustic cell. $\tau_{\mathrm{A}}$ is full width at half maximum (FWHM) of an auto correlation spectra of the reference signal, so that it should taken as a measure of the time resolution of the measuring system. In the present experiment, the factors which influence the limit of time resolution are the rotating speed of the chopper and the length of the M-series PRBS. Figure 4 shows the dependencies of $\tau_{0}, \tau_{\mathrm{f}}$ and $\tau_{\mathrm{A}}$ on various chopper speeds for the copper foil sample. $\tau_{0}$ is about 2 times as large as $\tau_{\mathrm{A}}$. This means the measured $\tau_{0}$ values are dependent on resolution of the measuring system. Consequently, precise measurement of the rising shape of impulse response is beyond the present experimental system. As seen in Fig. $4, \tau_{\mathrm{f}}$ and $\tau_{0}$ approach constant values with the difference about $17 \mathrm{~ms}$ between them. This difference is considered to show the net thermal

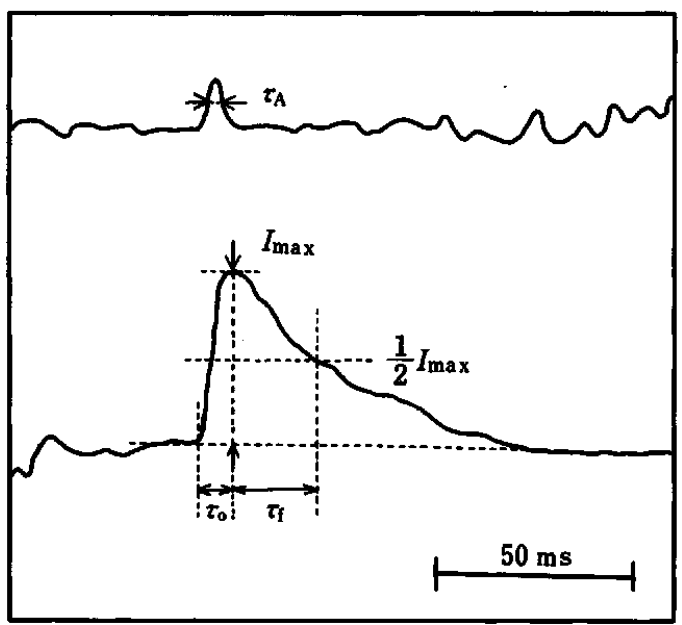

Fig. 3 The first kind correlation spectra of copper foil sample of $10 \mu \mathrm{m}$ thick(bottom). Top curve is the autocorrelation of reference signal. $\tau_{\mathrm{A}}, \tau_{0}, \tau_{\mathrm{f}}$ and $I_{\max }$ are defined in the text.

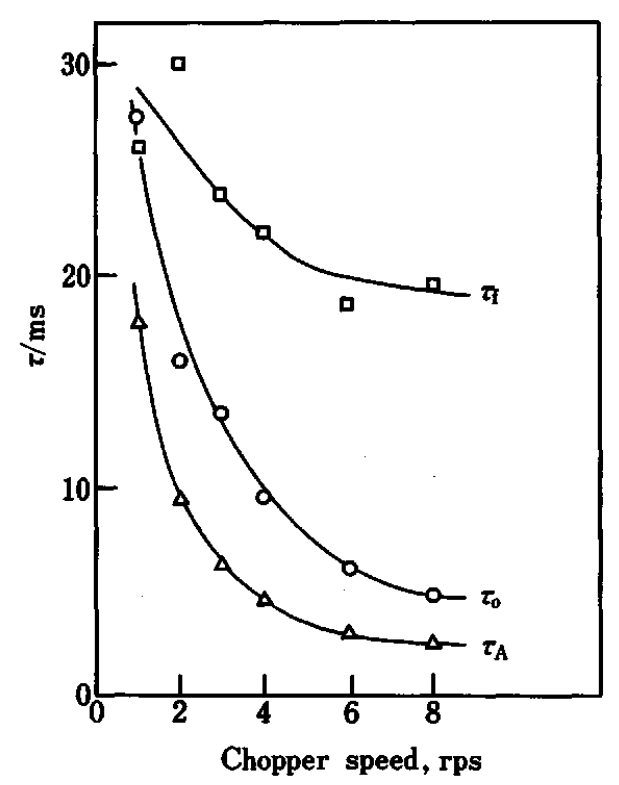

Fig. 4 Dependence of $\tau_{A}, \tau_{0}$ and $\tau_{f}$ on chopper speed for the copper foil sample of $10 \mu \mathrm{m}$ thick.

relaxation time of sample foil. Plots of signal intensity, $I_{\max }$, versus chopper speed are shown in Fig. $5 . I_{\max }$ is inversely proportional to the chopper speed; this is similar to the way that the photoacoustic signal in normal chopping shows inverse dependence on chopping frequency. This implies that an intense beam of the $S R$ is advantageous as an exciting source for attaining the high resolution of time.

Figure 6 shows the plots of $\tau_{\mathrm{f}}, \tau_{0}$ and $I_{\max }$ versus sample thickness at a constant chopper speed (4rps).

The $\tau_{0}$ and $\tau_{\mathrm{f}}$ show the maximum values at the sample thickness of 50 and $10 \mu \mathrm{m}$, respectively, and then decrease with the increase of the thickness. Optical 


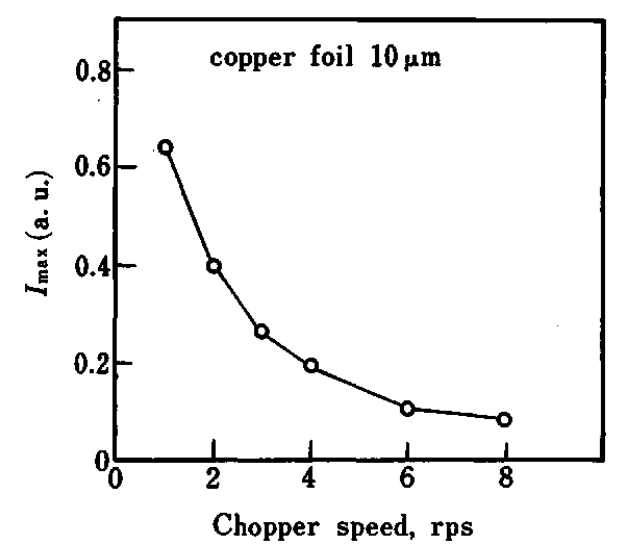

Fig. 5 Dependence of $I_{\max }$ of correlation spectra of copper foil sample of $10 \mu \mathrm{m}$ thick on chopper speed.

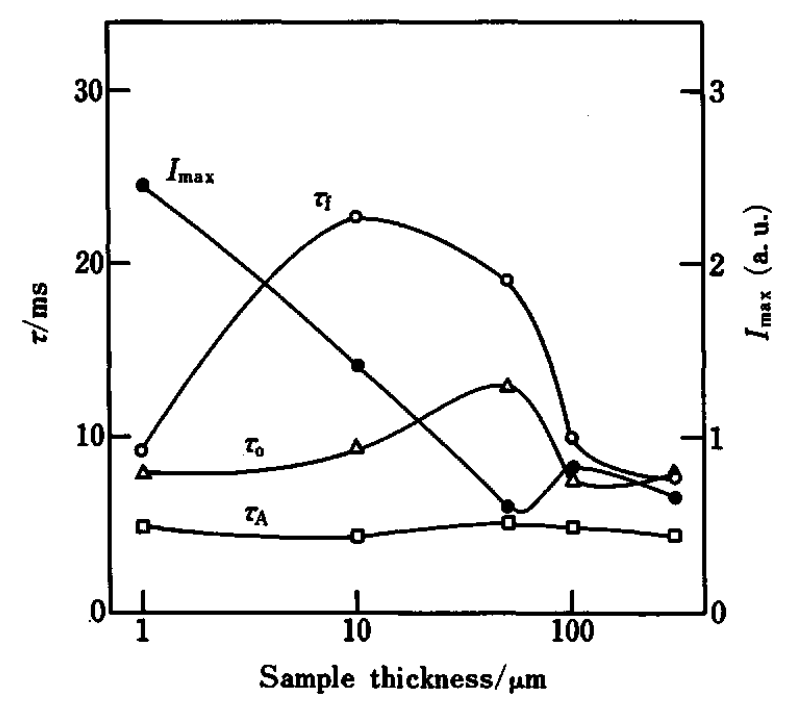

Fig. 6 Changes of $\tau_{0}, \tau_{\mathrm{f}}$ and $I_{\max }$ of correlation spectra of copper samples versus sample thickness.

penetration depth of copper for X-rays $(1.37 \AA)$ is calculated to be about $4 \mu \mathrm{m}$ from the values of mass absorption coefficient about $280 \mathrm{~cm}^{2} \mathrm{~g}^{-19}$ and density $8.93 \mathrm{~g} \mathrm{~cm}^{-3}$. The copper samples used in this experiment were optically thick except for the sample of $1 \mu \mathrm{m}$ thickness. In the samples with the thickness larger than the optical penetration depth, heat dissipation may occur in the process of diffusion, causing the rapid decrease of surface temperature. In the cases where the dissipation is neglected, $\tau_{\mathrm{f}}$ shows a monotonous change with the increase of the sample thickness, as seen in the case of the laminated sample (Fig. 7). The decrease of $\tau_{\mathrm{f}}$ for the thick sample of copper in the present experiment is probably due to the dissipation of heat in the sample. The decrease of $\tau_{0}$ value with the increase of sample thickness would be explained as follows: for the samples with the large $\tau_{0}$ value, the temperature of the sample surface is maintained relatively high for long period, so that the

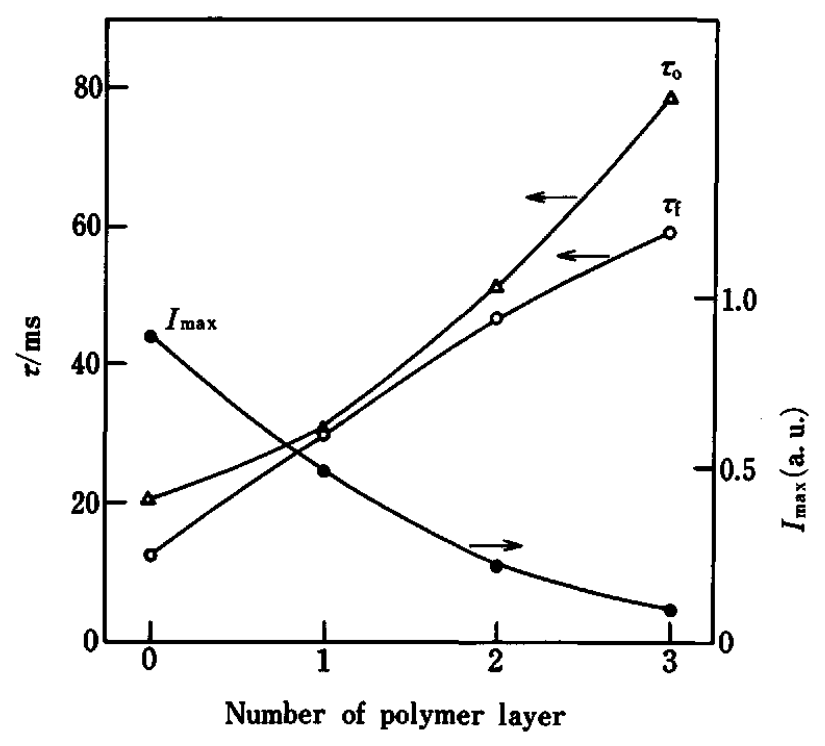

Fig. 7 Changes of $\tau_{0}, \tau_{\mathrm{f}}$ and $I_{\max }$ of laminated samples versus number of adhesive tapes on the substrate.

heat supply from surface to the air is continued for a long period, causing the increase of $\tau_{0}$. On the other hand, the small heat capacity for the sample $1 \mu \mathrm{m}$ thick explains the short $\tau_{\mathrm{f}}$ and large $I_{\max }$.

Figure 7 shows plots of $\tau_{0}, \tau_{\mathrm{f}}$ and $I_{\max }$ as a function of number of adhesive tape layers for laminated samples at constant chopper speed (4rps). $\tau_{0}$ and $\tau_{f}$ show monotonous increase with the number of tapes. The increased $\tau_{0}$ values shows the increased delay of the generated heat to diffuse to the surface of the sample through the tapes. The increased $\tau_{\mathrm{f}}$ and decreased $I_{\max }$ reflect the increased heat resistance between the copper layer and the inner atmosphere of the sample cell by adhering the tapes on the copper layer. Referring to the thermonics, the time constant $\tau_{\mathrm{t}}$ of thermal relaxation is calculated as $\tau_{t}=R C$, where $R$ and $C$ are heat resistance and heat capacity of the sample. ${ }^{10} . \tau_{1}$ is related to $\tau_{\mathrm{f}}$ as $\tau_{\mathrm{f}}=0.692 \tau_{\mathrm{t}}$. Therefore heat resistance and heat capacity can be easily obtained as $R=\tau_{\mathrm{f}} /$ $(0.692 C)$ and $C=\tau_{\mathrm{f}} /(0.692 R)$. In this experiment, $C$ can be given to be $3.45 \times 10^{-2} \mathrm{~J} \mathrm{~K}^{-1} \mathrm{~cm}^{-2}$ with the values of specific heat capacity $24.5 \mathrm{~J} \mathrm{~K}^{-1} \mathrm{~mol}^{-1}$, density $8.96 \mathrm{~g}$ $\mathrm{cm}^{-3}$, atomic weight $63.55 \mathrm{~g} \mathrm{~mol}^{-111}$, and thickness 0.01 $\mathrm{cm}$. $R$ values for laminated samples are calculated to be $0.51,1.25,1.95$ and $2.47 \mathrm{~J}^{-1} \mathrm{~K} \mathrm{~s} \mathrm{~cm}^{2}$ using the $\tau_{\mathrm{f}}$ values of 12.2, 29.8, 46.7 and $59.1 \mathrm{~ms}$. Thermal characteristics of surface can thus be easily measured using X-ray correlation photoacoustics.

\section{References}

1. A. Rosencwaig, "Photoacoustics and Photoacoustic Spectroscopy", John Wiley \& Sons, New York (1980).

2. S. Mascarennas, H. Vargas and C. L. Cesar, Med. Phys., 11, 73 (1984). 
3. R. Germer, Rev. Sci. Instrum., 55, 1461 (1984).

4. K. Kato, Y. Kobayashi, S. Aoki and Y. Sugitani, Anal. Sci, 3, 275 (1987).

5. T. Masujima, H. Kawata, Y. Amemiya, N. Kamiya, T. Katsura, T. Iwamoto, H. Yoshida, H. Imai, and M. Ando, Chem. Lett., 1987, 973.

6. Y. Sugitani, A. Uejima and K. Kato, J. Photoacoust., 1, 217 (1982).

7. A. Uejima and Y. Sugitani, J. Photoacoust., 1, 397 (1983).
8. G. F. Kirkbright, R. M. Miller and Y. Sugitani, Anal. Chem., 56, 2043 (1984).

9. C. H. Macgillary, G. D. Rieck and K. Lonsdale, "International Tables for X-ray Crystallography", Vol. 3, p. 175, Kynoch Press, Birmingham (1962).

10. H. S. Carslaw and J. C. Jaeger, "Conduction of Heat in Solids", 2nd ed., Oxford Clarendon Press, Oxford (1953).

11. “Rikagaku, Jiten", 4th ed., Iwanami Shoten, Tokyo (1987).

(Received December 21, 1987) (Accepted January 12, 1988) 